\title{
IMPACT OF WEATHER VARIABLES ON ELECTRICITY POWER DEMAND FORECAST USING FUZZY LOGIC TECHNIQUE
}

\author{
S. A. Benson ${ }^{1}$ and J. K. Ogunjuyigbe ${ }^{2, *}$ \\ 1,2, DEPARTMENT OF ELECTRICAL ENGINEERING, UNIVERSITY OF ABUJA, ABUJA, NIGERIA \\ E-mail addresses: ${ }^{1}$ iamsteveben@gmail.com, ${ }^{2}$ k_oguns@yahoo.com
}

\begin{abstract}
Fuzzy logic, being a very robust artificial intelligence technique, was employed to forecast short term load in this work. The algorithm was demonstrated with the help of seven months average daily load data collected from Power Holding Company of Nigeria (PHCN). The outcome realized from the model or simulation illustrate that the projected forecasting technique, which propose the use of weather alteration such as temperature and humidity, gives load forecasting results with substantial precision, in the range of 0.71\% Mean Absolute Percentage Error (MAPE). Using the conventional technique provided forecast with a large MAPE up to $3.18 \%$ which shows a high level of inaccuracy or less precision as compared with the use of Fuzzy logic.
\end{abstract}

Keywords: Temperature, Humidity, Fuzzy Logic, Autoregressive.

\section{INTRODUCTION}

Load forecasting has played a fundamental role in the resourceful planning, processing or operation and maintenance or protection of power systems. Short term load forecasting is essential for the control and preparation and or scheduling operation of a power system and also serves as input to the power analysis function such as load flow, eventuality and contingency analysis. The analysis made on the monthly load and monthly weather statistics helps in understanding the alterations which may affect load forecasting. As stated by [1], "Euclidean norm weight factors are used to evaluate the similarity between the forecast day and the searched previous day. Euclidean norm makes us comprehend the similarity by using the expression based on idea and concept of norm. The new Euclidean norm uses maximum temperature, average humidity and day type with weight factor to evaluate the similarity of the searched previous day". The expression for the new Euclidean norm is as follows:

$$
\begin{gathered}
\mathrm{EN}=\left[\mathrm{W}_{1}(\mathrm{dTMAX})^{2}+\mathrm{W}_{2}(\mathrm{dHavg})^{2}+\mathrm{W}_{3}(\mathrm{dD})^{2}\right]^{1 / 2} \\
\mathrm{dT}_{\max }=\mathrm{T}_{\max }-\mathrm{TP}_{\max } \\
\mathrm{dH}_{\text {avg }}=\mathrm{H}_{\text {avg }}-\mathrm{HP}_{\text {avg }} \\
\mathrm{dD}=\mathrm{D}-\mathrm{Dp}
\end{gathered}
$$

Where $\mathrm{T}_{\max }$ and $\mathrm{H}_{\text {avg }}$ are the forecast day maximum temperature and average humidity respectively.
Likewise, $\mathrm{Tp}_{\max }$ and $\mathrm{Hp}_{\text {avg }}$ are the maximum temperature and average humidity of the searched previous day and $\mathrm{W}_{1}, \mathrm{~W}_{2}$ and $\mathrm{W}_{3}$ are the weight factor ascertained by least square method based on the regression model constructed using past statistics. The load forecast at any given time not only rely on the load of the preceding time but also on the load at the given time on the preceding day.

However, the fuzzy inference system is used to estimate the similarity between the preceding forecast day and preceding similar days ensuing in rectification or correction factor, used to correct the similar days of the forecast day to achieve the load forecast [1].

Although propose fuzzy based medium and long term forecasting has been demonstrated on a comparatively smaller scale or magnitude. But, the algorithm is capable of forecasting load with very good accuracy. All it needs is a rigorous study of the load statistics or data, factor affecting the load statistics and feature accountable for errors in the forecast value of load. Results obtained by proposed fuzzy logic method based long term forecasting; prove better-quality or superior to conservative and or conventional techniques in forecasting medium and long term load [2]. This usually proposed fuzzy-based forecast works in two levels, this implies, training and on-line forecasting. The training stage, involves fine tuning process which define the appropriate rules that will give an optimal 
expected value. This training process is repeated so that the input parameter gives an output with minimal error. The metered past load information and data with a one-minute period resolution are first passed via a low-pass filter which in this case, the membership function to sieve out the rapidly varying unsystematic component. In [6] the filter statistics are then used to train a $2 \mathrm{x}$-input, $2 \mathrm{y}$-output fuzzy logic-based forecaster to create pattern data base by using the first-order and second-order difference of the data. After enough training, it will be linked with a controller to foretell the load change on-line (ie providing data from existing power system on real time since the training stage is often done using existing data). To generate the pattern data base and rules, appropriate fuzzy membership functions are first defined for input data and output data respectively. Once the pattern is built, it is ready to work. [3]

"In the stochastic time-series method, the load is modeled as the output of a linear filter driven by white noise" [4]. Based on the distinctiveness of the linear filter, dissimilar load models can be formulated. The autoregressive (AR) and moving average (MA) set of procedures are the two basic types of stochastic time series, and while neither of these processes is usually independently capable of accurately modeling the load, they form the basis for development of more complex processes.

In the AR process, the current value of load is uttered linearly in terms of preceding values and a random noise. The orders of this process rely on the oldest previous value for which the load is regressed. The moving average process, on the other hand, expresses the load linearly in terms of current and previous values of a white noise series, and again the order of the series depends on the oldest previous value.

"The autoregressive and moving average processes are usually combined to give the popular autoregressive moving average (ARMA) process, which has found widespread use in the power industry. In the ARMA process, the load at any instant is expressed as a linear combination of its past values and a white noise series. The order of this process is specified by the order of the AR and MA series included in its composition. Time series used for AR, MA, or ARMA models are referred to as stationary processes when their means and covariance are stationary with respect to time. So if the process being modeled is non-stationary, it is first transformed to a stationary series before being modeled by the AR, MA, or ARMA process". [5]

The recognition of the stochastic time-series move toward online forecasting arises mainly from the point of accuracy obtainable and its simplicity of online implementation. The identification process of the timeseries models is a major disadvantage because the process requires extensive analysis of raw load data through the use of range mean, correlation, and autocorrelation analysis.

Therefore complimenting the field of loads forecast, this research has chosen to add effort to the mode of forecasting. Since previous works have been limited to certain sphere of the forecast such as hourly and daily forecast for the short term load forecasting, this study will center on forecast of average weekly (similar days) load forecast. This is because, it will provide a collective means of addressing the amount of load that will be aim at being consumed (i.e. giving an estimated load required for consumption in a given week as against the hourly and daily forecast). Applying fuzzy logic to power system, this research will improvise by modifying the model of forecasting that will yield result and at the same time user friendly.

\section{MATERIALS AND METHODS}

The historic daily load demand data from power holding company of Nigeria (PHCN) was collected for the month of February to August and the load outline is studied and analyzed. Based on the analysis, a load forecasting model is then developed using fuzzy logic method. MATLAB software is used extensively in the project for modeling and analysis of the load outline in order to forecast the load demand accurately.

The ensuing forecasted data after step of modification is then justified by evaluating the forecasting error present to the actual load. The other analysis includes calculating the absolute error (AE). High error indicates a less robust model design with a small or low accuracy. The errors are calculated using the formula as follows:

$$
\begin{aligned}
& R E=\frac{\text { forecast loads }- \text { actual load }}{\text { actual load }} \times 100 \% \\
& A E=\frac{\text { forecast load }- \text { actual load }}{\text { actual load }} \times 100 \%
\end{aligned}
$$

As for the relative error (RE) or comparative error, a positive value of error shows an over forecast, which indicates or means that the forecast is larger than the actual load. A negative value on the other hand indicates under forecast, where the forecasted load value is less than the actual value. The modification process must be repeated until a suitable Mean Absolute Percentage Error (MAPE) is achieved. The flow chart below describes the process of using the fuzzy logic method. 


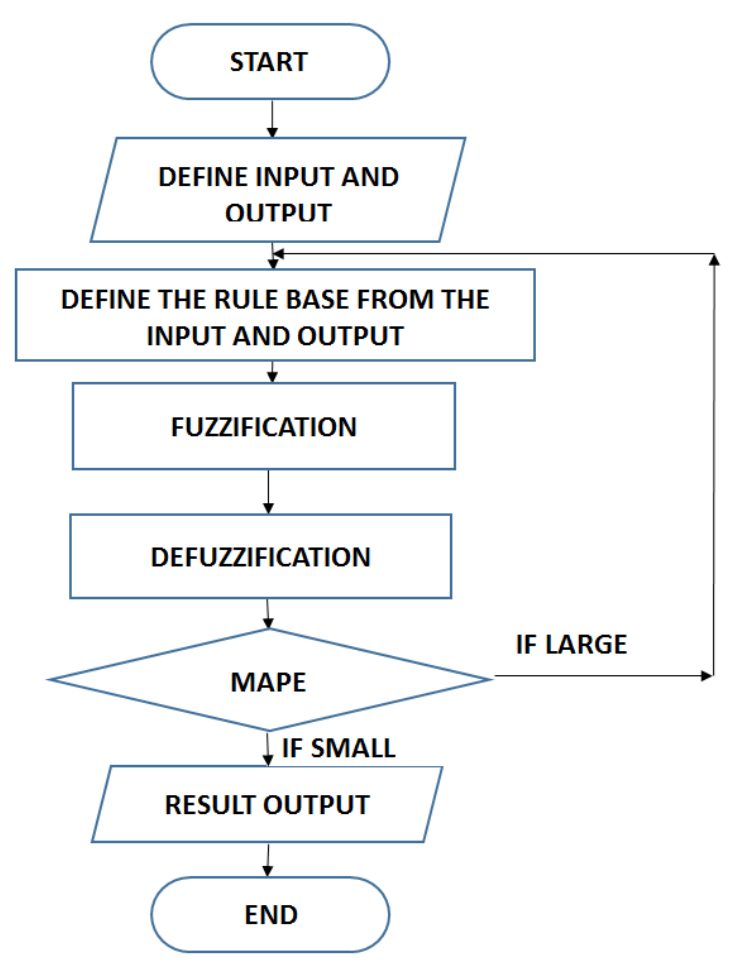

Figure1: Flow chart depicting load forecasting algorithm using fuzzy logic.

\section{RESULTS AND DISCUSSION}

The performance of the technique for the short term load forecast is tested by using the seven months data of average weekly similar days, that is, from February to August. The technique has been simulated using the fuzzy logic toolbox obtainable in MATLAB. Load forecasting is done for the month of August. Hence, the statistics or data of the month of July has been used for the selection of similar days. The number of similar days used for the forecasting is seven. This same data set are been used to test the convention technique as well in other to demonstrate the superiority of fuzzy logic.

The parameters of the fuzzy membership functions are determined through the simulation of the load curve forecasting in the previous month to the forecast day. The parameters of the membership functions for the input and output variables for the month of August similar days average weekly load curve forecasting are as follows. In Tables 1,2 and 3 which gives the membership function input of the fuzzy interference system although graphical user interface can be used to illustrate or symbolize the membership function. Without these membership function input providing the pathway for simulation, the fuzzy interference remain void.

The projected model is tested to forecast the average weekly load demand. The forecast experiments are simulated for Monday through to Sunday. One month average weekly forecast is determined and the MAPE is calculated to represent the forecast accuracy. The forecasting of load using linear regression show high level of discrepancy from the actual load; thus making it less efficient in forecasting when compared to fuzzy logic given the deviation from the actual value of load.

\begin{tabular}{l|l}
$\begin{array}{l}\text { Table 1: Parameter of the membership function of the } \\
\text { input variable(temperature) }\end{array}$ \\
\hline \multirow{3}{*}{ MONDAY } & $\mathrm{T}_{\mathrm{L}} 0,5,10$ \\
& $\mathrm{~T}_{\mathrm{N}} 8,15.5,23$ \\
& $\mathrm{~T}_{\mathrm{H}} 20,25,30$ \\
\hline \multirow{4}{*}{ TUESDAY } & $\mathrm{T}_{\mathrm{L}} 0,4.67,9.33$ \\
& $\mathrm{~T}_{\mathrm{N}} 7.47,14.47,21.47$ \\
& $\mathrm{~T}_{\mathrm{H}} 18.4,23.2,28$ \\
\hline \multirow{5}{*}{ WEDNESDAY } & $\mathrm{T}_{\mathrm{L}} 0,2,6$ \\
& $\mathrm{~T}_{\mathrm{N}} 4,11,18$ \\
& $\mathrm{~T}_{\mathrm{H}} 13,18,27$ \\
\hline \multirow{5}{*}{ THURSDAY } & $\mathrm{T}_{\mathrm{L}} 0,3.5,7$ \\
& $\mathrm{~T}_{\mathrm{N}} 5,12,19$ \\
& $\mathrm{~T}_{\mathrm{H}} 14,20,27$ \\
\hline \multirow{5}{*}{ FRIDAY } & $\mathrm{T}_{\mathrm{L}} 0,4,8$ \\
& $\mathrm{~T}_{\mathrm{N}} 6,13,20$ \\
& $\mathrm{~T}_{\mathrm{H}} 15,21.5,28$ \\
\hline \multirow{3}{*}{ SATURDAY } & $\mathrm{T}_{\mathrm{L}} 0,3.71,7$ \\
& $\mathrm{~T}_{\mathrm{N}} 5.57,12.07,18.57$ \\
& $\mathrm{~T}_{\mathrm{H}} 13.93,19.96,26$ \\
\hline \multirow{3}{*}{ SUNDAY } & $\mathrm{T}_{\mathrm{L}} 0,5,10$ \\
& $\mathrm{~T}_{\mathrm{N}} 8,15.5,23$ \\
& $\mathrm{~T}_{\mathrm{H}} 20,25,30$ \\
\hline
\end{tabular}

Table 2: Parameter of the membership function of the input variable (humidity)

\begin{tabular}{|c|c|}
\hline MONDAY & $\begin{array}{ccccc}\mathrm{H}_{\mathrm{L}} & 10.99, & 19.00, & 21.00, & 29.00 \\
\mathrm{H}_{\mathrm{N}} & 26.25, & 36.25, & 38.75, & 48.75 \\
\mathrm{H}_{\mathrm{H}} & 41.79, & 56.21, & 59.8, & 74.2 \\
\end{array}$ \\
\hline TUESDAY & $\begin{array}{lllll}\mathrm{H}_{\mathrm{L}} & 15.00, & 23.00, & 25.00, & 33.00 \\
\mathrm{H}_{\mathrm{N}} & 30.25, & 40.25, & 42.75, & 52.75 \\
\mathrm{H}_{\mathrm{H}} & 45.8, & 60.20, & 63.80, & 78.20\end{array}$ \\
\hline WEDNESDAY & $\begin{array}{lllll}\mathrm{H}_{\mathrm{L}} & 11.03, & 19.27, & 21.33, & 29.58 \\
\mathrm{H}_{\mathrm{N}} & 26.74, & 37.05, & 39.62, & 49.92 \\
\mathrm{H}_{\mathrm{N}} & 42.76, & 57.6, & 61.31, & 76.15 \\
\end{array}$ \\
\hline THURSDAY & $\begin{array}{lllll}\mathrm{H}_{\mathrm{L}} 16.01, & 24.13, & 26.16, & 34.29 \\
\mathrm{H}_{\mathrm{N}} 31.49, & 41.65, & 41.81, & 54.33 \\
\mathrm{H}_{\mathrm{H}} 47.28, & 61.9, & 65.56, & 80.18 \\
\end{array}$ \\
\hline FRIDAY & $\begin{array}{ccccc}\mathrm{H}_{\mathrm{L}} & 15.95, & 23.86, & 25.83, & 33.71 \\
\mathrm{H}_{\mathrm{N}} & 31.00, & 40.85, & 43.31, & 53.16 \\
\mathrm{H}_{\mathrm{H}} & 46.31, & 60.51, & 64.05, & 78.23\end{array}$ \\
\hline SATURDAY & $\begin{array}{llllc}\mathrm{H}_{\mathrm{L}} 16.98, & 24.86, & 26.83, & 34.71 \\
\mathrm{H}_{\mathrm{N}} 32.00, & 41.85, & 44.31, & 54.16 \\
\mathrm{H}_{\mathrm{H}} & 47.31, & 61.51, & 65.05, & 79.23\end{array}$ \\
\hline SUNDAY & $\begin{array}{llll}\mathrm{H}_{\mathrm{L}} & 9.00, & 19.00, & 29.00 \\
\mathrm{H}_{\mathrm{N}} & 24.00, & 26.50, & 49.00 \\
\mathrm{H}_{\mathrm{H}} & 49.00, & 57.00, & 75.00\end{array}$ \\
\hline
\end{tabular}


Table 3: Parameters of the Membership Function of the Output Variable

\begin{tabular}{l|l}
\hline \multirow{3}{*}{ MONDAY } & $2844,2860,2876$ \\
& $2864,2880,2896$ \\
& $2884,2900,2916$ \\
\hline \multirow{3}{*}{ TUESDAY } & $2762,2800,2838$ \\
& $2810,2848,2886$ \\
& $2857,2895,2933$ \\
\hline \multirow{3}{*}{ WEDNESDAY } & $2840,2860,2880$ \\
& $2865,2820,2874$ \\
& $2890,2910,2930$ \\
\hline \multirow{3}{*}{ FHURSDAY } & $2766,2820,2874$ \\
& $2833,2887,2941$ \\
& $2900,2954,3008$ \\
\hline \multirow{3}{*}{ SATURDAY } & $2858,2890,2922$ \\
& $2898,2930,2922$ \\
\hline SUNDAY & $2936,2970,3002$ \\
\hline
\end{tabular}

Table 4: similar days average weekly forecasted and actual electricity load demand for the month of august 2010

\begin{tabular}{llll}
\hline Days & $\begin{array}{l}\text { Forecast } \\
\text { using linear } \\
\text { regression } \\
\text { (KWATT) }\end{array}$ & $\begin{array}{l}\text { Forecast } \\
\text { using fuzzy } \\
\text { logic } \\
\text { (KWATT) }\end{array}$ & $\begin{array}{l}\text { Actual Load } \\
\text { (KWATT) }\end{array}$ \\
\hline Monday & 2808.20 & 2880 & 2880 \\
Tuesday & 2791.41 & 2847.5 & 2900 \\
Wednesday & 2798.44 & 2885 & 2880 \\
Thursday & 2777.81 & 2887 & 2870 \\
Friday & 2785.94 & 2930 & 2970 \\
Saturday & 2784.69 & 2795 & 2820 \\
Sunday & 2805.08 & 2740 & 2735 \\
\hline
\end{tabular}

Table 5 Table showing Absolute Relative Error and MAPE

\begin{tabular}{lcc}
\hline DAYS & $\begin{array}{c}\text { Absolute Relative } \\
\text { Error using Linear } \\
\text { Regression }\end{array}$ & $\begin{array}{c}\text { Absolute } \\
\text { Relative Error } \\
\text { using Fuzzy } \\
\text { Logic }\end{array}$ \\
\hline MONDAY & 2.49 & 0 \\
TUESDAY & 3.74 & 1.81 \\
WEDNESDAY & 2.83 & 0.17 \\
THURSDAY & 3.21 & 0.59 \\
FRIDAY & 6.19 & 1.35 \\
SATURDAY & 1.25 & 0.89 \\
SUNDAY & 2.56 & 0.18 \\
\hline
\end{tabular}

With the projected methods, the MAPE error for the considered similar days, for which forecasted results are obtained and shown as in Table 4 are calculated for both linear regression as 3.18 and for fuzzy procedure as.0.71.

\section{CONCLUSION AND RECOMMENDATION}

The outcome realized from the simulation illustrate that the projected forecasting methodology, which proposes the use of weather alteration or variables i.e. temperature and humidity, gives load forecasting results with substantial accuracy, in the range of $0.71 \%$ MAPE. Using the conventional technique provided forecast with a large MAPE up to $3.18 \%$ which shows a high level of inaccuracy or less precision as compared with the use of Fuzzy logic. Therefore, the proposed methodology proves to be superior in forecast and will be more obliging in using more weather variables, which will certainly be better than using only temperature as the weather variable affecting the load as used in the conventional technique in short term load forecasting.

\section{REFERENCES}

[1] Srinivas, E. and A. Jain, "A Methodology for Short Term Load Forecasting using Fuzzy Logic and Similarity", The National Conference on Advances in Computational Intelligence Applications in Power, Control, Signal Processing and Telecommunications (NCACI-2009), Bhubaneswar, India, March 20-22, 2009, pp. 2-4.

[2] Pujar, J., "Fuzzy Ideology Based Long Term Load Forecasting", World academy of science, Engineering and Technology, Vol 64, 2010, pp. 636638.

[3] Liu, K., Kwan, C., Lewis, F., Subbarayan, S., Shoults, R. and Naccarino, J, "Comparison of very Short Term Load Forecasting Techniques", IEEE Transaction on Power System, vol. 11, Number 2, 1996, pp. 878-880.

[4] Drezga, I., Rahman, S., "Short Term Load Forecasting with Local ANN Predictor," IEEE Transaction on Power System, Vol 14, Number 3, 1999, pp. 844850.

[5] Willis, H., Finley, L., Buri, M.,"Forecasting Electricity Demand of Distribution System in Rural and Sparsely Populated Region", IEEE Transaction on Power System, Vol 10, 1995, pp. 2008-2013.

[6] Liu, K., Kwan, C., Lewis, F .L, Subbarayan, S., Shoults, R. R. and Naccarino, J., "Comparison of very Short Term Load Forecasting Techniques", IEEE Transaction on Power System, vol. 11, Number 2, 1996, pp. 878-880.

[7] Ekemezie, P. N. and Osuagwu C. C., "A SelfOrganizing Fuzzy Logic Control", Nigerian Journal of Technology, Vol 30, Number 1, 2001, pp. 1-9.

[8] Musa S.Y., Mbaga, E.V., "Daily Nigerian Peak Load Forecasting using Artificial Neural Network with Seasonal Indices", Nigerian Journal of Technology, Vol 33, Number 1, 2014, pp. 114-118. 